\title{
Soluzione salina ipertonica e furosemide ad alte dosi nella sindrome cardiorenale: esperienza personale
}

\author{
Hypertonic saline solution and high-dose furosemide infusion in cardiorenal \\ syndrome: our experience
}

\section{Francesco Ventrella*, Sergio Cappello, Graziano Minafra, Maria Pipino, Mattea Carbone, Maria Insalata, Luigi Caccetta, Luigi lamele}

Struttura Complessa di Medicina interna (Direttore: dr Francesco Ventrella), ASL Foggia, Presidio Ospedaliero "Giuseppe Tatarella" Cerignola, Foggia

Ricevuto il 4 febbraio 2011; accettato il 5 maggio 2011

disponibile online l'1 luglio 2011

\section{KEYWORDS \\ Heart failure; \\ Renal failure; \\ Hypertonic saline \\ solution; \\ Furosemide; \\ Cardiorenal syndrome.}

\begin{abstract}
Summary
Introduction: Heart failure is frequently complicated by renal failure, and this association is a negative prognostic factor. These patients sometimes present oligo-/anuria and resistance to high-dose furosemide, a condition referred to as the cardiorenal syndrome (CRS). Acute or chronic reductions in left ventricular function result in decreased blood flow, with reduction of renal perfusion and activation of several neurohormonal systems, which cause resistance to diuretic therapy. This condition often requires ultrafiltration, which is an effective, but invasive and expensive procedure. Infusions of hypertonic saline solution (HSS) and high-dose furosemide can be an effective alternative.

Materials and methods: From November 2009 through May 2010, our team treated 20 patients with CRS and resistance to iv boluses of high-dose furosemide. These patients were treated with small-volume (150-250 mL) infusions of $\mathrm{HSS}$ ( $\mathrm{NaCl} 1.57-4.5 \%$, depending on serum $\mathrm{Na}$ values) and high-dose furosemide twice a day. The aim of this treatment is to modify renal hemodynamics and the water-saline balance in the kidney by counteracting the extracellular fluid accumulation and eliminating symptoms of congestion.

Results: In 18 patients (90\%), urine output was restored and renal function improved during the first hours of treatment. Clinical improvement was evident from the first day of therapy, and there were no adverse events. Two patients $(10 \%)$ did not respond to the treatment: one (who had been in critical condition since admission) died; the other required regular sessions of ultrafiltration.
\end{abstract}

\footnotetext{
* Corrispondenza: via Garibaldi 23 - 76017 San Ferdinando di Puglia (BT).

E-mail: f.ventrella@tiscali.it (F. Ventrella).
} 
Conclusions: HSS combined with high-dose furosemide is a safe, effective, low-cost approach to the treatment of CRS that is resistant to diuretic therapy.

(c) 2011 Elsevier Srl. All rights reserved.

\section{Introduzione}

Le funzioni di cuore e rene sono strettamente interconnesse al fine di mantenere l'omeostasi dei fluidi extracellulari, sia nel soggetto sano sia in presenza di alterata funzione di uno dei due organi, come descritto già diversi decenni fa dal celebre fisiologo A.G. Guyton [1].

Lo studio delle interazioni tra le funzioni cardiaca e renale si è sviluppato nel corso degli ultimi decenni, sottolineando l'importanza ora dell'uno, ora dell'altro apparato a seconda dell'area culturale di provenienza dei gruppi di ricerca. Le importanti connessioni cardiorenali descritte da Guyton sono state recentemente "rivisitate" [2] e si è giunti a una ben definita delineazione della Sindrome Cardiorenale (SCR), condizione fisiopatologica in cui l'insufficienza di uno dei due apparati ha ripercussioni sull'altro, con esito finale nel peggioramento della prognosi.

Ancora più recentemente si è giunti a una classificazione unitaria della SCR in 5 tipi $[3,4]$ (tab. 1).

Argomento del presente articolo sono le prime due forme, di più frequente riscontro nella pratica clinica dei reparti di Medicina Interna. Infatti, lo scompenso cardiaco acuto o cronico spesso si complica con l'insufficienza renale; tale associazione costituisce un fattore prognostico negativo più importante della gravità clinica (secondo le classi definite dalla New York Heart Association, NYHA) e della valutazione ecocardiografica della funzionalità sistolica del cuore [5].

La diagnosi di sindrome cardiorenale può essere formulata in presenza di insufficienza cardiaca sistolica o diastolica, contrazione della diuresi, edema e versamenti trasudatizi, nonché presenza di disfunzione renale, definita come incremento della creatininemia $\geq 0,3 \mathrm{mg} \%$ e/o riduzione di 9$15 \mathrm{~mL} / \mathrm{min}$ della velocità di filtrazione glomerulare (VFG) rispetto ai valori precedenti [6]. Spesso la SCR si associa a iponatriemia.

Il trattamento della ritenzione idrosalina in corso di SCR costituisce tuttora un problema complesso e non universalmente definito [7].

Elemento essenziale che condiziona la cattiva prognosi di tale sindrome è la comparsa di una refrattarietà alla terapia con diuretici dell'ansa, che ancora oggi rappresentano il cardine del trattamento: tali pazienti presentano oligo-anuria anche in corso di somministrazione di elevati dosaggi di furosemide (500-1.000 mg/die endovena).

Partendo dall'assunto che i diuretici rimangono essenziali per il trattamento dello scompenso cardiaco, il gruppo palermitano di Licata ha ideato, sperimentato e proposto una soluzione biologicamente plausibile per migliorare e/o ripristinare la risposta diuretica nella SCR $[6,8-11]$. Si tratta della somministrazione di un piccolo volume $(150 \mathrm{~mL})$ di soluzione salina ipertonica di $\mathrm{NaCl}$ (SSI) (dall'1,4\% al 4,6\%), con l'aggiunta di 20-40 mEq di $\mathrm{KCl}+$ furosemide ad alte dosi $(125-1.000 \mathrm{mg})$, da infondere endovena in 30 minuti 2 volte al giorno, secondo un protocollo standardizzato, differenziato sulla scorta dei valori di natriemia:
- se la natriemia è $<125 \mathrm{mEq} / \mathrm{L}$, la SSI sarà al 4,6\%;

- se la natriemia è compresa tra 126 e 135 mEq/L, la SSI sarà al 3,5\%;

- se la natriemia è $>135 \mathrm{mEq} / \mathrm{L}$, la SSI sarà all'1,4-2,4\%.

Tale trattamento si associa a una dieta standard con 120 mmol di sodio e $100 \mathrm{mmol}$ di potassio, restrizione idrica ( $1 \mathrm{~L} / \mathrm{die}$ ) e al trattamento farmacologico di base dell'insufficienza cardiaca con inibitori dell'enzima di conversione dell'angiotensina (ACE-inibitori), sartani, vasodilatatori, digitale, beta-bloccanti, antiaggreganti o anticoagulanti [6].

Il protocollo si pone come possibile alternativa al trattamento che, dai dati della letteratura, risulta essere il gold standard in questi pazienti, cioè l'ultrafiltrazione, tecnica sottrattiva molto efficace, soprattutto nei pazienti anasarcatici, fortemente iponatriemici e con grave e progressiva insufficienza renale, che è in grado di rimuovere velocemente l'eccesso di liquidi e consentire, spesso, un drastico miglioramento dell'emodinamica cardiaca e della responsività renale $[12,13]$. L'ultrafiltrazione è tuttavia una procedura invasiva, di costo elevato e richiede personale esperto e dedicato; sarebbe perciò auspicabile adottarla come trattamento di seconda linea nei pazienti non altrimenti gestibili con la terapia diuretica [14].

Gli autori dichiarano che lo studio presentato è stato realizzato in accordo con gli standard etici stabiliti nella

Tabella 1 Classificazione attuale della sindrome cardiorenale.

- Tipo 1: sindrome cardiorenale acuta. È la più comune ed è caratterizzata da un rapido peggioramento della funzione cardiaca che conduce a ipoperfusione del rene e conseguente danno renale

- Tipo 2: sindrome cardiorenale cronica. È caratterizzata dalla presenza di insufficienza cardiaca cronica con ipoperfusione e congestione venosa renale cronica e conseguente danno progressivo al rene

- Tipo 3: sindrome renocardiaca acuta. È caratterizzata da un brusco e primitivo peggioramento della funzione renale, con conseguente danno cardiaco acuto

- Tipo 4: Sindrome renocardiaca cronica. Condizione primaria di insufficienza renale cronica che contribuisce alla riduzione della funzione cardiaca, alla disfunzione diastolica e all'ipertrofia ventricolare sinistra

- Tipo 5: sindrome cardiorenale secondaria. È caratterizzata dalla presenza di insufficienza cardiaca e disfunzione renale, a causa di patologie sistemiche acute o croniche (lupus eritematoso sistemico, amiloidosi, sepsi ecc.), che colpiscono primitivamente entrambi gli organi

Fonti: Ronco C, et al. J Am Coll Cardiol 2008;52(19):1527-39; Iwanaga Y, et al. Circ J 2010;74(7):1274-82. 
Dichiarazione di Helsinki, e che il consenso informato è stato ottenuto da tutti i partecipanti prima del loro arruolamento allo studio.

\section{Materiali e metodi}

Partendo dai presupposti sopra descritti, abbiamo effettuato uno studio osservazionale di esperienza sul campo su una piccola popolazione di 20 pazienti con sindrome cardiorenale, ricoverati nella nostra UO nel periodo novembre 2009maggio 2010. Per le sue caratteristiche, lo studio non ha certo l'obiettivo di fornire conclusioni inconfutabili in merito all'efficacia del protocollo adottato, ma si propone di valutare l'applicabilità, la maneggevolezza e l'utilità "nel mondo reale" del trattamento con SSI + furosemide ad alte dosi nella gestione dei pazienti con SCR, ricoverati in una UO di Medicina interna di un ospedale di livello intermedio.

La diagnosi di SCR è stata formulata sulla base dei seguenti criteri:

- diagnosi clinica di scompenso cardiaco congestizio con congestione polmonare e dispnea, edemi declivi e/o versamenti sierosi;

- presenza di disfunzione renale, definita come incremento della creatininemia $\geq 0,3 \mathrm{mg} \%$ e/o riduzione di $9-15 \mathrm{~mL} / \mathrm{min}$ della VFG (calcolata mediante la formula di Cockcroft e Gault) rispetto ai valori precedenti, insorta ex novo oppure in un paziente già portatore di insufficienza renale cronica di minor grado;

- contrazione della diuresi ( $\leq 800 \mathrm{~mL} /$ die) dopo almeno 24 ore di terapia con furosemide endovena a dose $\geq 250 \mathrm{mg} /$ die, quale segno di refrattarietà alla terapia diuretica.

Nove pazienti erano di sesso maschile, 11 di sesso femminile; l'età media era di 77,5 anni (età minima 57 anni, età massima 88 anni). Quasi tutti i pazienti erano ipotesi (pressione arteriosa media 94/59 $\pm 10 \mathrm{mmHg}$ ); il 75\% era iponatriemico $(\mathrm{Na}<135 \mathrm{mEq} / \mathrm{L})$.

L'entità dell'insufficienza renale era rilevante: creatininemia $2,2 \pm 0,8 \mathrm{mg} \%$, velocità di filtrazione glomerulare (VFG) pari a $28 \pm 14 \mathrm{~mL} / \mathrm{min}$. Secondo la classificazione KDOQI dell'insufficienza renale, i pazienti erano inquadrabili nei seguenti stadi di gravità:

- Stadio 1 (VFG $\geq 90 \mathrm{~mL} / \mathrm{min}) 0$ pazienti

- Stadio 2 (VFG $60-89 \mathrm{~mL} / \mathrm{min}) 1$ paziente (5\%)

- Stadio 3 (VFG 59-30 mL/min) 6 pazienti (30\%)

- Stadio 4 (VFG 29-15 mL/min) 13 pazienti (65\%)

- Stadio 5 (VFG $<15 \mathrm{~mL} / \mathrm{min}) 0$ pazienti.

La diuresi presente dopo 24 di terapia con furosemide in bolo endovena, a dosi $\geq 250 \mathrm{mg} /$ die, era di $550 \pm 166 \mathrm{~mL} /$ $24 \mathrm{~h}$.

L'eziologia della cardiopatia di base era quasi equamente distribuita tra la forma ipertensiva (7 pazienti), quella ischemica (7 pazienti) e quella dilatativa (5 pazienti); un solo paziente presentava un'eziologia valvolare (stenosi aortica).

Tabella 2 Caratteristiche cliniche dei pazienti selezionati.

\begin{tabular}{|c|c|c|c|c|c|c|c|c|c|c|}
\hline Paziente $\mathrm{N}$. & Sesso & $\begin{array}{c}\text { Età } \\
\text { (anni) }\end{array}$ & $\begin{array}{l}\text { Peso } \\
(\mathrm{kg})\end{array}$ & $\begin{array}{l}\text { Creatinina } \\
(\mathrm{mg} \%)\end{array}$ & $\begin{array}{c}\text { VFG } \\
(\mathrm{mL} / \mathrm{min})\end{array}$ & $\begin{array}{c}\text { Azotemia } \\
\text { (mg\%) }\end{array}$ & $\begin{array}{l}\text { Sodiemia } \\
(\mathrm{mEq} / \mathrm{L})\end{array}$ & $\begin{array}{l}\text { Diuresi } \\
(\mathrm{mL} / 24 \mathrm{~h})\end{array}$ & $\begin{array}{c}\mathrm{PA} \\
(\mathrm{mmHg})\end{array}$ & $\begin{array}{c}\text { Eziologia } \\
\text { cardiopatia }\end{array}$ \\
\hline 1 & $\mathrm{~F}$ & 78 & 52 & 1,20 & 32 & 95 & 132 & 500 & $90 / 60$ & Dilatativa \\
\hline 2 & $\mathrm{~F}$ & 84 & 84 & 3,17 & 18 & 170 & 124 & 250 & $90 / 50$ & Ischemica \\
\hline 3 & $\mathrm{~F}$ & 80 & 71 & 2,50 & 20 & 146 & 129 & 500 & $90 / 60$ & Ipertensiva \\
\hline 4 & $\mathrm{~F}$ & 87 & 62 & 2,00 & 19 & 160 & 133 & 500 & $90 / 60$ & Stenosi aortica \\
\hline 5 & $\mathrm{~F}$ & 85 & 56 & 2,50 & 15 & 158 & 134 & 500 & $90 / 60$ & Ipertensiva \\
\hline 6 & $M$ & 83 & 88 & 3,10 & 22 & 97 & 120 & 700 & $80 / 50$ & Ischemica \\
\hline 7 & M & 87 & 73 & 2,20 & 24 & 87 & 133 & 250 & $80 / 40$ & Dilatativa \\
\hline 8 & $M$ & 57 & 90 & 1,40 & 74 & 73 & 130 & 600 & $110 / 80$ & Ipertensiva \\
\hline 9 & M & 73 & 60 & 1,30 & 43 & 50 & 136 & 800 & $120 / 80$ & Ischemica \\
\hline 10 & $\mathrm{~F}$ & 76 & 53 & 1,40 & 29 & 100 & 130 & 600 & $90 / 60$ & Ischemica \\
\hline 11 & $M$ & 80 & 75 & 2,80 & 22 & 154 & 125 & 800 & $100 / 60$ & Ipertensiva \\
\hline 12 & $\mathrm{~F}$ & 68 & 69 & 3,00 & 20 & 180 & 126 & 500 & $95 / 60$ & Dilatativa \\
\hline 13 & $\mathrm{~F}$ & 75 & 70 & 1,80 & 30 & 150 & 139 & 800 & $90 / 50$ & Ipertensiva \\
\hline 14 & $M$ & 80 & 66 & 1,50 & 37 & 140 & 128 & 500 & $80 / 60$ & Dilatativa \\
\hline 15 & $M$ & 75 & 90 & 3,20 & 25 & 195 & 121 & 700 & $95 / 50$ & Ischemica \\
\hline 16 & $M$ & 88 & 84 & 3,50 & 17 & 210 & 124 & 300 & $100 / 50$ & Dilatativa \\
\hline 17 & $\mathrm{~F}$ & 60 & 48 & 1,00 & 45 & 50 & 135 & 600 & $110 / 70$ & Ipertensiva \\
\hline 18 & $\mathrm{~F}$ & 79 & 72 & 2,50 & 21 & 160 & 128 & 500 & $90 / 60$ & Ischemica \\
\hline 19 & $M$ & 75 & 70 & 1,80 & 35 & 170 & 140 & 700 & $100 / 60$ & Ipertensiva \\
\hline 20 & $\mathrm{~F}$ & 80 & 69 & 2,50 & 20 & 165 & 135 & 500 & $90 / 60$ & Ischemica \\
\hline
\end{tabular}

Legenda: VFG = velocità di filtrazione glomerulare; $\mathrm{PA}=$ pressione arteriosa. 
Le principali caratteristiche cliniche dei pazienti selezionati sono riportate nella tab. 2 .

Tutti i pazienti assumevano ACE-inibitori o sartani e/o antialdosteronici. I beta-bloccanti, se già in terapia prima del ricovero, venivano proseguiti, altrimenti si attendevano almeno 2-3 giorni di stabilità clinica prima di iniziarne la somministrazione al minimo dosaggio iniziale.

I pazienti, prima di essere proposti alla valutazione del nefrologo per l'eventuale ultrafiltrazione, sono stati sottoposti a trattamento con SSI e furosemide, secondo il protocollo riportato nella tab. 3 .

Rispetto al protocollo originale del gruppo di Licata [6] sopra citato, abbiamo adottato alcune modifiche, per ragioni di praticità riguardo all'applicazione degli schemi nell'ordinaria organizzazione del nostro reparto.

- La scelta del grado di ipertonicità è stata effettuata sulla base della natriemia del paziente, secondo il seguente schema:

- natriemia $\geq 135 \mathrm{mEq} / \mathrm{L} \rightarrow$ soluzione $\mathrm{NaCl}$ al 1,57-2,17\%;

- natriemia 130-134 $\mathrm{mEq} / \mathrm{L} \rightarrow$ soluzione $\mathrm{NaCl}$ al 2,7\%;

- natriemia 125-129 $\mathrm{mEq} / \mathrm{L} \rightarrow$ soluzione $\mathrm{NaCl}$ al 3,6\%;

- natriemia $<125 \mathrm{mEq} / \mathrm{L} \rightarrow$ soluzione $\mathrm{NaCl}$ al 4,5\%.

- L'aggiunta di $\mathrm{KCl}$ è stata effettuata solo nei pazienti con kaliemia $\leq 4,5 \mathrm{mEq} / \mathrm{L}$, utilizzando fiale di $\mathrm{KCl}$ da $20 \mathrm{mEq}$ in $10 \mathrm{~mL}$, in modo da contenere la concentrazione di $\mathrm{KCl}$ nella soluzione $\leq 10 \mathrm{mEq} / 100 \mathrm{~mL}$, in ottemperanza al protocollo adottato nella nostra Azienda per la regolamentazione dell'uso delle soluzioni concentrate di potassio, emanato ai sensi della Raccomandazione del Ministero della Salute n. 1 del marzo 2008 [15], secondo il seguente schema:

- se la kaliemia era $<3,6 \mathrm{mEq} / \mathrm{L}$, si aggiungeva 1 fiala di $\mathrm{KCl}$;

- se la kaliemia era compresa tra 3,6 e 4,4 mEq/L, si aggiungeva $1 / 2$ fiala di $\mathrm{KCl}$.

Tabella 3 Protocollo terapeutico per l'uso di SSI + furosemide nella sindrome cardiorenale utilizzato presso la nostra Unità Operativa.

Soluzione salina ipertonica di $\mathrm{NaCl}$ a diversa ipertonicità in base alla natriemia

- Natriemia $\geq 135 \mathrm{mEq} / \mathrm{L}$ : soluzione all'1,57-2,17\% (fisiologica $150 \mathrm{~mL}+1-2$ fiale $\mathrm{NaCl} 20 \mathrm{mEq}$ in $10 \mathrm{~mL}$ )

- Natriemia 130-134 mEq/L: soluzione al 2,7\% (fisiologica $150 \mathrm{~mL}+3$ fiale $\mathrm{NaCl} 20 \mathrm{mEq}$ in $10 \mathrm{~mL}$ )

- Natriemia 125-129 mEq/L: soluzione al 3,6\% (fisiologica $150 \mathrm{~mL}+5$ fiale $\mathrm{NaCl} 20 \mathrm{mEq}$ in $10 \mathrm{~mL}$ )

- Natriemia < $125 \mathrm{mEq} / \mathrm{L}$ : soluzione al 4,5\% (fisiologica $100 \mathrm{~mL}+5$ fiale $\mathrm{NaCl} 20 \mathrm{mEq}$ in $10 \mathrm{~mL}$ )

$+$ furosemide ad alte dosi (1-4 fiale da $250 \mathrm{mg}$ )

$+$

se kaliemia $\leq 4,5 \mathrm{mEq} / \mathrm{L}$, aggiunta di $\mathrm{KCl}$ in fiale da $20 \mathrm{mEq}$ in $10 \mathrm{~mL}$

- 1 fiala intera se kaliemia $<3,6 \mathrm{mEq} / \mathrm{L}$

$-1 / 2$ fiala se kaliemia tra 3,6 e 4,4 mEq/L

Protocollo modificato rispetto a quello originale di Licata $\mathrm{G}$, et al. Intern Emerg Med 2009;4:S104-14.
Alla soluzione ipertonica è stata aggiunta inizialmente una fiala di furosemide da $250 \mathrm{mg}$. Se dopo 24 ore la diuresi giornaliera era $<1.000 \mathrm{~mL}$, la dose della furosemide veniva progressivamente incrementata fino a un massimo di $1.000 \mathrm{mg}$.

Il trattamento è stato effettuato 2 volte al giorno, infondendo la soluzione in vena nell'arco di 30 minuti.

Nei pazienti con pressione sistolica $<100 \mathrm{mmHg}$, si è solitamente associata l'infusione di dopamina a "dosaggi renali" $(2-5 \mathrm{mcg} / \mathrm{kg} / \mathrm{min})$, iniziata almeno 24 ore prima dell'avvio del trattamento con SSI.

\section{Risultati}

Nella tab. 4 sono riportati, per ciascun paziente, lo schema terapeutico adottato (grado di ipertonicità della SSI e dosaggio giornaliero della furosemide) e i risultati ottenuti con il trattamento, in termini di variazione dei principali parametri clinici e di laboratorio prima e dopo il ciclo di terapia, con indicazione dei giorni di trattamento effettuati.

Sul totale dei 20 pazienti trattati, nel 90\% (18 pazienti) si è ottenuta una risposta favorevole con ripresa della diuresi, miglioramento della funzione renale e del quadro clinico; per 2 pazienti, invece, la terapia non si è dimostrata efficace ( fig. 1). La durata media del trattamento è stata di 8 giorni.

Nella tab. 5 sono riportate media e deviazione standard dei principali parametri clinici e di laboratorio dei 20 pazienti, prima e dopo il trattamento (creatininemia, azotemia, natriemia, kaliemia, diuresi giornaliera, VFG, pressione arteriosa sistolica e diastolica) con valutazione della significatività statistica delle variazioni.

La diuresi è nettamente aumentata, da $555 \pm 166$ a $3.215 \pm 1.252 \mathrm{~mL} / 24 \mathrm{~h}$. La creatininemia e la VFG sono sensibilmente migliorate passando, rispettivamente, da $2,2 \pm 0,8 \mathrm{mg} \%$ e $28 \pm 14 \mathrm{~mL} / \mathrm{min}$ prima del trattamento a $1,58 \pm 0,7 \mathrm{mg} \%$ e $42 \pm 22 \mathrm{~mL} / \mathrm{min}$ al termine del ciclo terapeutico. Anche i valori di natriemia hanno mostrato una tendenza al miglioramento (da $130 \pm 6 \mathrm{mEq} / \mathrm{L}$ prima a $140 \pm 4 \mathrm{mEq} / \mathrm{L}$ dopo), con progressiva correzione dell'iponatriemia, tanto frequente prima del trattamento.

Per quanto riguarda la velocità della risposta al trattamento, nei 18 pazienti responder la ripresa della diuresi si è ottenuta fin dalle prime 24 ore di trattamento, per poi stabilizzarsi nei giorni successivi. Il miglioramento dei parametri di funzionalità renale è stato, ovviamente, graduale nei giorni successivi.

L'applicazione del test $t$ di Student ha dimostrato la significatività statistica delle variazioni di tutti i parametri esaminati, prima e dopo il trattamento, a eccezione della kaliemia (tab. 5).

Da segnalare, infine, che non si sono presentati effetti collaterali significativi. Non si è avuto calo dei valori pressori rispetto ai bassi valori di partenza; anzi, alla fine della terapia, la pressione arteriosa si è stabilizzata a livelli più vicini alla normalità rispetto a quella presente prima del trattamento. L'aggiunta alle SSI di 10-20 mEq di $\mathrm{KCl}$, prevista dal protocollo nei pazienti con valori di kaliemia $\leq 4,4 \mathrm{mEq} / \mathrm{L}$, ha prevenuto 0 corretto l'ipokaliemia da terapia diuretica.

Solo in 2 pazienti (10\%) il trattamento non ha avuto successo:

- la paziente n. 4 non ha avuto alcun miglioramento ed è andata incontro a exitus dopo 6 giorni dall'inizio della 
Tabella 4 Dosaggi adottati e principali parametri clinici e di laboratorio, prima e dopo il trattamento, nei singoli pazienti.

\begin{tabular}{|c|c|c|c|c|c|c|c|c|c|c|c|c|c|c|c|c|c|c|}
\hline \multirow[t]{2}{*}{$\begin{array}{l}\text { Paziente } \\
\text { N. }\end{array}$} & \multirow[t]{2}{*}{$\begin{array}{l}\text { SSI } \\
(\% \mathrm{NaCl})\end{array}$} & \multirow[t]{2}{*}{$\begin{array}{l}\text { Furosemide } \\
\text { (mg/die) }\end{array}$} & \multicolumn{2}{|c|}{$\begin{array}{l}\text { Creatinina } \\
(\mathrm{mg} \%)\end{array}$} & \multicolumn{2}{|c|}{$\begin{array}{l}\text { Azotemia } \\
\text { (mg\%) }\end{array}$} & \multicolumn{2}{|c|}{$\begin{array}{l}\text { Sodiemia } \\
(\mathrm{mEq} / \mathrm{L})\end{array}$} & \multicolumn{2}{|c|}{$\begin{array}{l}\text { Kaliemia } \\
\text { (mEq/L) }\end{array}$} & \multicolumn{2}{|c|}{$\begin{array}{l}\text { Diuresi } \\
\text { (cc/24 h) }\end{array}$} & \multicolumn{2}{|c|}{$\begin{array}{l}\text { VFG } \\
(\mathrm{mL} / \mathrm{min})\end{array}$} & \multicolumn{2}{|c|}{$\begin{array}{l}\text { Pressione } \\
\text { arteriosa } \\
(\mathrm{mmHg})\end{array}$} & \multirow[t]{2}{*}{$\begin{array}{l}\text { Durata } \\
\text { terapia } \\
\text { (giorni) }\end{array}$} & \multirow[t]{2}{*}{ Risultato } \\
\hline & & & Prima & Dopo & Prima & Dopo & Prima & Dopo & Prima & Dopo & Prima & Dopo & Prima & Dopo & Prima & Dopo & & \\
\hline 1 & 2,7 & 500 & 1,2 & 0,8 & 95 & 61 & 132 & 147 & 3,7 & 3,8 & 500 & 3.000 & 32 & 48 & $90 / 60$ & $110 / 70$ & 10 & Favorevole \\
\hline 2 & 4,5 & 500 & 3,17 & 1,5 & 170 & 107 & 124 & 147 & 2,7 & 3,3 & 250 & 4.300 & 18 & 37 & $90 / 50$ & $115 / 60$ & 7 & Favorevole \\
\hline 3 & 3,6 & 1.000 & 2,5 & 2,1 & 146 & 157 & 129 & 137 & 2,9 & 3,1 & 500 & 3.900 & 20 & 24 & $90 / 60$ & $120 / 70$ & 8 & Favorevole \\
\hline 4 & 2,7 & 500 & 2,0 & 3,3 & 160 & 190 & 133 & 139 & 4,1 & 4,3 & 500 & 700 & 19 & 12 & $90 / 60$ & - & 6 & Decesso \\
\hline 5 & 2,7 & 500 & 2,5 & 1,1 & 158 & 77 & 134 & 143 & 4,8 & 4,2 & 500 & 4.700 & 15 & 33 & $90 / 60$ & $110 / 60$ & 7 & Favorevole \\
\hline 6 & 4,5 & 250 & 3,1 & 2,1 & 97 & 38 & 120 & 136 & 5,1 & 4,2 & 700 & 3.500 & 22 & 33 & $80 / 50$ & $110 / 60$ & 9 & Favorevole \\
\hline 7 & 2,7 & 500 & 2,2 & 3,3 & 87 & 115 & 133 & 135 & 3,9 & 3,6 & 250 & 900 & 24 & 16 & $80 / 40$ & $100 / 60$ & $\begin{array}{l}12+ \\
8 \mathrm{UF}^{*}\end{array}$ & Ultrafiltrazione \\
\hline 8 & 2,7 & 250 & 1,4 & 0,9 & 28 & 26 & 130 & 138 & 2,7 & 3,7 & 600 & 5.500 & 74 & 115 & $110 / 80$ & $130 / 80$ & 8 & Favorevole \\
\hline 9 & 2,17 & 500 & 1,3 & 1,2 & 50 & 45 & 136 & 132 & 4 & 3,5 & 800 & 2.500 & 43 & 47 & $120 / 80$ & $100 / 60$ & 6 & Favorevole \\
\hline 10 & 2,7 & 500 & 1,4 & 0,9 & 100 & 58 & 130 & 145 & 3,5 & 3,9 & 600 & 2.800 & 29 & 44 & $90 / 60$ & $110 / 60$ & 8 & Favorevole \\
\hline 11 & 3,6 & 500 & 2,8 & 1,9 & 154 & 106 & 125 & 136 & 4,2 & 4,6 & 800 & 3.200 & 22 & 33 & $100 / 60$ & $110 / 70$ & 9 & Favorevole \\
\hline 12 & 3,6 & 500 & 3,0 & 1,8 & 180 & 100 & 126 & 140 & 2,8 & 3,5 & 500 & 3.000 & 20 & 33 & $95 / 60$ & $100 / 60$ & 8 & Favorevole \\
\hline 13 & 2,17 & 250 & 1,8 & 1,0 & 150 & 76 & 139 & 145 & 2,9 & 3,6 & 800 & 2.500 & 30 & 54 & $90 / 50$ & $110 / 60$ & 6 & Favorevole \\
\hline 14 & 3,6 & 500 & 1,5 & 0,9 & 140 & 68 & 128 & 136 & 3 & 3,5 & 500 & 3.000 & 37 & 61 & $80 / 60$ & $95 / 60$ & 8 & Favorevole \\
\hline 15 & 4,5 & 500 & 3,2 & 1,8 & 195 & 120 & 121 & 138 & 3,5 & 4 & 700 & 3.500 & 25 & 45 & $95 / 50$ & $110 / 70$ & 9 & Favorevole \\
\hline 16 & 4,5 & 500 & 3,5 & 2,1 & 210 & 125 & 124 & 140 & 4,5 & 4,2 & 300 & 2.000 & 17 & 21 & $100 / 50$ & $100 / 60$ & 9 & Favorevole \\
\hline 17 & 2,17 & 500 & 1,0 & 0,8 & 50 & 40 & 135 & 145 & 2,7 & 3,6 & 600 & 5.500 & 45 & 57 & $110 / 70$ & $120 / 80$ & 8 & Favorevole \\
\hline 18 & 3,6 & 500 & 2,5 & 1,8 & 160 & 104 & 128 & 139 & 3 & 3,8 & 500 & 3.000 & 21 & 29 & $90 / 60$ & $100 / 60$ & 9 & Favorevole \\
\hline 19 & 1,57 & 500 & 1,8 & 1,1 & 170 & 85 & 140 & 145 & 3,5 & 4 & 700 & 2.800 & 35 & 57 & $100 / 60$ & $125 / 70$ & 7 & Favorevole \\
\hline 20 & 2,17 & 500 & 2,5 & 1,1 & 165 & 80 & 135 & 140 & 4,2 & 4,3 & 500 & 4.000 & 20 & 44 & $90 / 60$ & $110 / 60$ & 7 & Favorevole \\
\hline
\end{tabular}

terapia. Si trattava di una donna di 87 anni, affetta da stenosi valvolare aortica severa, con importante comorbilità dovuta a insufficienza respiratoria cronica di vecchia data. Peraltro la stessa paziente, per le sue gravi condizioni di partenza, non è stata ritenuta dai nefrologi eleggibile al trattamento con ultrafiltrazione;

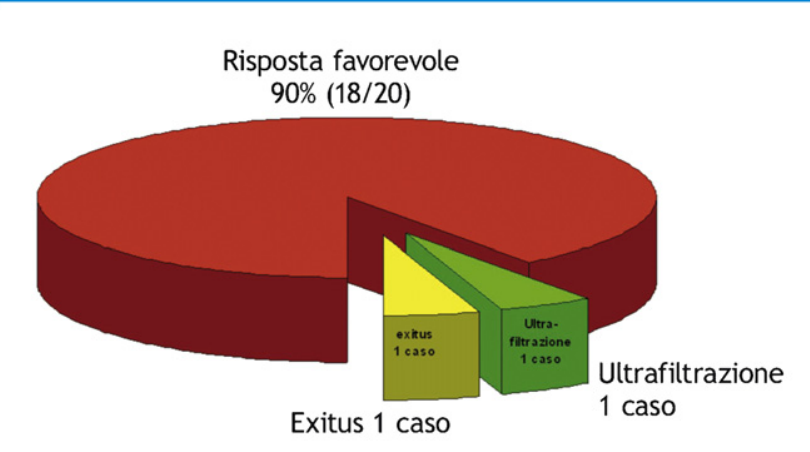

Figura 1 Risultati della nostra esperienza personale nell'uso di SSI + furosemide ad alte dosi nel trattamento della ritenzione idrosalina nella sindrome cardiorenale (20 pazienti in totale, trattati da novembre 2009 a maggio 2010).
- il paziente n. 7, di anni 87, affetto da cardiopatia dilatativa e fortemente ipoteso, ha risposto scarsamente al trattamento, non riuscendo a conseguire la stabilizzazione del quadro clinico. Per questo motivo, dopo 12 giorni di terapia con SSI + furosemide, è stato sottoposto a ultrafiltrazione per 8 giorni, trattamento che gli ha permesso di raggiungere la stabilità clinica e quindi di passare alla terapia di mantenimento per os.

\section{Discussione}

Sebbene la terapia diuretica della congestione da insufficienza cardiaca sia in uso da oltre 40 anni, in letteratura sono presenti pochi dati di evidence-based medicine, e le linee guida esistenti sul tema sono frutto prevalentemente di opinioni di esperti. Ne consegue che, nonostante l'impiego molto frequente dei diuretici nella comune pratica medica, è tuttora in atto, in letteratura, un dibattito su quali siano le migliori strategie di somministrazione dei diuretici dell'ansa, in particolare in ordine al dosaggio (alte o basse dosi) e alle modalità di somministrazione (bolo endovena o infusione continua) [16-18].

In tale dibattito può ben inserirsi l'opzione dell'uso della furosemide ad alte dosi, associata all'infusione di SSI, che è stata oggetto della nostra esperienza. 
Tabella 5 Media e deviazione standard dei principali parametri clinici e di laboratorio prima e dopo il trattamento. Valutazione statistica con test $t$ di Student.

\begin{tabular}{lccc}
\hline & Prima & Dopo & P \\
\hline Creatininemia (mg\%) & $2,2 \pm 0,8$ & $1,58 \pm 0,7$ & $<0,01$ \\
\hline Azotemia (mg\%) & $133 \pm 51$ & $89 \pm 41$ & $<0,004$ \\
\hline Natriemia (mEq/L) & $130 \pm 6$ & $140 \pm 4$ & $<0,0001$ \\
\hline Kaliemia (mEq/L) & $3,6 \pm 0,7$ & $3,8 \pm 0,4$ & $<0,1$ (non significativo) \\
\hline Diuresi (mL/24h) & $555 \pm 166$ & $3.215 \pm 1.252$ & $<0,0001$ \\
\hline VFG (mL/min) & $28 \pm 14$ & $42 \pm 22$ & $<0,02$ \\
\hline PA sistolica (mmHg) & $94 \pm 10$ & $110 \pm 9$ & $<0,0001$ \\
\hline PA diastolica (mmHg) & $59 \pm 10$ & $65 \pm 7$ & $<0,04$ \\
\hline Durata terapia (giorni) & $8 \pm 1,5$ & &
\end{tabular}

Legenda: VFG = velocità di filtrazione glomerulare; $\mathrm{PA}=$ pressione arteriosa.

Diversi meccanismi sono stati ipotizzati per spiegare l'azione terapeutica di quest'associazione, che è stata definita "non convenzionale e controintuitiva" [19].

A nostro giudizio, il razionale di tale approccio va ricondotto essenzialmente a due effetti principali, che verranno brevemente analizzati di seguito [9]:

- aumento della volemia efficace e, quindi, della perfusione renale;

- aumento della concentrazione intratubulare di sodio.

Il substrato fisiopatologico del deficit progressivo della funzione renale nella SCR è la riduzione della gittata cardiaca, con ipoperfusione degli organi del circolo sistemico, e del rene in particolare, e attivazione delle risposte sistemiche di feedback (sistema nervoso simpatico, sistema reninaangiotensina-aldosterone, altri sistemi neurormonali) [2]. Tale risposta neurormonale è spesso destinata al fallimento, poiché, a causa dell'eccessiva vasocostrizione e del consistente passaggio di fluidi dal compartimento intravascolare a quello interstiziale, in molti casi si determina un'ulteriore progressiva riduzione del flusso plasmatico renale e, pertanto, una contrazione del filtrato glomerulare e della diuresi e un incremento della creatininemia. L'infusione della SSI aumenta rapidamente la pressione osmotica plasmatica e quindi, richiamando osmoticamente liquidi nel comparto intravascolare, espande la volemia e incrementa il flusso renale. Questo effetto favorisce direttamente l'aumento del filtrato glomerulare e di conseguenza la ripresa della diuresi; inoltre, incrementa il trasporto ematico della furosemide a livello del nefrone, suo sito di azione.

La furosemide, oltre a determinare una significativa venodilatazione sistemica, con importante riduzione del precarico [20], agisce direttamente sul nefrone attraverso il blocco di una proteina di trasporto delle cellule della branca ascendente dell'ansa di Henle, il simporto (symporter) $\mathrm{Na}^{+}-\mathrm{K}^{+}-2 \mathrm{Cl}^{-}$ [21]. Ne consegue riduzione del riassorbimento tubulare di $\mathrm{NaCl}$, nonché notevole aumento della sua escrezione urinaria e della quantità di acqua osmoticamente corrispondente. Inoltre, il blocco del riassorbimento attivo di $\mathrm{NaCl}$ interferisce con un passaggio critico della formazione dell'ambiente ipertonico della midollare del rene, e quindi del "meccanismo a controcorrente", alla base della capacità del rene di concentrare le urine. I diuretici dell'ansa, pertanto, impediscono al rene di concentrare le urine e, perciò, causano diuresi ipotonica, favorendo l'eliminazione anche di acqua libera [21].

Da quanto esposto si comprende perché l'iponatriemia, spesso presente nella SCR, per la quale rappresenta un potente predittore di mortalità [22], possa contribuire alla riduzione dell'effetto farmacologico della furosemide e quindi all'insorgenza della "refrattarietà alla terapia diuretica". L'apporto sodico dovuto all'infusione delle SSI, aumentando la concentrazione intratubulare di sodio, substrato per l'azione della furosemide, può contribuire a restituirle efficacia.

Si potrebbe obiettare che, tra le diverse forme eziopatogenetiche di iponatriemia [23], quella presente nello scompenso cardiaco è tipicamente un'iponatriemia ipervolemica, che si connota non per una carenza del pool totale di sodio dell'organismo (che, anzi, può essere aumentato per l'iperaldosteronismo secondario tipico dello scompenso cardiaco), ma piuttosto per una ritenzione di acqua con conseguente iponatriemia da diluizione.

Tuttavia, nei pazienti con insufficienza cardiaca in stadio avanzato, che fanno uso cronico (e a volte eccessivo) di diuretici dell'ansa e sono a dieta iposodica, si può determinare una reale riduzione del pool sodico totale dell'organismo, anche perché il trattamento farmacologico di base dello scompenso cardiaco cronico, con ACE-inibitori o sartani e antialdosteronici, blocca, più o meno intensamente, la funzione sodio-ritentiva dell'aldosterone, correggendo l'iperaldosteronismo secondario.

In sintesi, nelle forme di grave scompenso cardiaco tipiche della SCR possono venire a mancare le due condizioni necessarie per ottenere una valida risposta alla terapia diuretica: un'adeguata quantità di sodio nel liquido intratubulare, disponibile per l'azione farmacodinamica del diuretico, e un'ottimale concentrazione e distribuzione del diuretico nel suo sito di azione (il tubulo renale) [24-27]. L'infusione delle SSI tende a correggere tali alterazioni.

Le prime dimostrazioni di efficacia di tale trattamento sono venute dai due trial clinici controllati italiani, realizzati dal gruppo palermitano di Licata, pubblicati rispettivamente nel 2000 e nel $2003[9,10]$, a cui si è aggiunto un terzo studio dello stesso gruppo di ricercatori, pubblicato nel 2005, che ha dimostrato, oltre alla buona risposta diuretica, anche una più rapida riduzione dei livelli di Brain Natriuretic Peptide (BNP) con le SSI + furosemide rispetto alla furosemide da sola [28]. 
Nella letteratura scientifica internazionale, nell'ambito del dibattito attualmente ancora in corso [29] su quali siano le migliori strategie e modalità di utilizzo dei diuretici dell'ansa nell'insufficienza cardiaca acuta, si registrano ulteriori segnalazioni sull'uso dell'associazione delle SSI + furosemide:

- una recente metanalisi della Cochrane Collaboration [30] ha valutato, fra i trial rientranti nei rigorosi standard sperimentali sull'argomento, anche uno degli studi italiani del gruppo di Licata [10] di confronto tra le SSI + furosemide vs furosemide da sola, che dimostra il vantaggio del primo protocollo;

- la Canadian Cardiovascular Society, nella consensus conference del 2007, cita l'infusione simultanea di SSI + furosemide quale opzione per ripristinare la diuresi e minimizzare il rischio di iponatriemia, sia pure come strategia non estesamente utilizzata rispetto all'ultrafiltrazione [31].

I nostri dati sono in linea con tali osservazioni e confermano l'applicabilità e la maneggevolezza di questo trattamento, anche al di fuori di un setting assistenziale di tipo intensivo o semintensivo. Per di più, segnaliamo che la gravità clinica dei pazienti del nostro studio, in termini sia di insufficienza renale sia di ipotensione arteriosa, era significativamente maggiore rispetto a quella dei soggetti arruolati nei trial sopra citati del gruppo palermitano $[9,10,28]$.

\section{Conclusioni}

La nostra esperienza, in quanto derivante da uno studio osservazionale di pratica clinica sul campo e su un piccolo campione di pazienti, non consente certamente di trarre conclusioni definitive sull'efficacia del protocollo di terapia adottato. Tuttavia, quanto da noi segnalato vuol contribuire a richiamare l'attenzione degli internisti su un semplice approccio terapeutico che, nei pazienti con sindrome cardiorenale e refrattarietà alla terapia diuretica, a un costo estremamente contenuto e senza effetti collaterali di rilievo, potrebbe rappresentare un utile tentativo di ripristinare la risposta alla terapia diuretica, prima di ricorrere alla tecnica di ultrafiltrazione; quest'ultima rimane attualmente il trattamento di riferimento, ma è invasiva e costosa, nonché più congeniale ad altri specialisti (nefrologi, cardiologi intensivisti).

Per tale ragione riteniamo auspicabile, nell'ambito della Medicina Interna, l'attuazione di trial clinici controllati e randomizzati, con un ampio campione di pazienti, da realizzare in maniera multicentrica, al fine di confermare con forte valenza sperimentale la validità di questo approccio terapeutico. Peraltro, questa nuova strategia di implementazione della terapia diuretica appare, ai nostri occhi di internisti, particolarmente affascinante nella sua semplicità, poiché si basa sull'utilizzo accorto di elementi primordiali, quali l'acqua, costituente fondamentale del nostro organismo, e il sodio, che ne rappresenta lo "scheletro osmotico", e ne sfrutta l'effetto sull'osmolarità e la volemia [32]. In sintesi, si avvale dei costituenti di quel "mare che è in noi" sul quale con ammirabile efficacia ci ha ripetutamente sensibilizzati il nostro collega Sgambato [33].

\section{Conflitto di interesse}

Gli autori dichiarano di essere esenti da conflitto di interessi.

\section{Bibliografia}

[1] Guyton AG. Trattato di fisiologia umana. Padova: Piccin; 1977.

[2] Bongartz LG, Cramer MJ, Doevendans PA, Joles JA, Braam B. The severe cardiorenal syndrome: 'Guyton revisited'. Eur Heart J 2005;26(1):11-7.

[3] Ronco C, Haapio M, House AA, Anavekar N, Bellomo R. Cardiorenal syndrome. J Am Coll Cardiol 2008;52(19):1527-39.

[4] Iwanaga Y, Miyazaki S. Heart failure, chronic kidney disease, and biomarkers-An integrated viewpoint. Circ J 2010;74(7):127482.

[5] Liang KV, Williams AW, Greene EL, Redfield MM. Acute decompensated heart failure and the cardiorenal syndrome. Crit Care Med 2008;36(1 Suppl):S75-88.

[6] Licata G. La sindrome cardiorenale. Nuovi approcci terapeutici. Intern Emerg Med 2009;4:S104-14.

[7] Nodari S, Palazzuoli A. Current treatment in acute and chronic cardio-renal syndrome. Heart Fail Rev 2010 [Epub ahead of print].

[8] Paterna S, Parrinello G, Amato P, Dominguez L, Pinto A, Maniscalchi T, et al. Tolerability and efficacy of high-dose furosemide and small-volume hypertonic saline solution in refractory congestive heart failure. Adv Ther 1999;16(5):219-28.

[9] Paterna S, Di Pasquale P, Parrinello G, Amato P, Cardinale A, Follone $\mathrm{G}$, et al. Effects of high-dose furosemide and smallvolume hypertonic saline solution infusion in comparison with a high dose of furosemide as a bolus, in refractory congestive heart failure. Eur J Heart Fail 2000;2(3):305-13.

[10] Licata G, Di Pasquale P, Parrinello G, Cardinale A, Scandurra A, Follone $G$, et al. Effects of high-dose furosemide and smallvolume hypertonic saline solution infusion in comparison with a high dose of furosemide as bolus in refractory congestive heart failure: long-term effects. Am Heart J 2003;145(3):459-66.

[11] Paterna S, Gaspare P, Fasullo S, Sarullo FM, Di Pasquale P. Normal-sodium diet compared with low-sodium diet in compensated congestive heart failure: is sodium an old enemy or a new friend? Clin Sci (Lond) 2008;114(3):221-30.

[12] Costanzo MR, Guglin ME, Saltzberg MT, Jessup ML, Bart BA, Teerlink JR, et al., UNLOAD Trial Investigators. Ultrafiltration versus intravenous diuretics for patients hospitalized for acute decompensated heart failure. J Am Coll Cardiol 2007;49(6): 675-83.

[13] Bart BA. Treatment of congestion in congestive heart failure: ultrafiltration is the only rational initial treatment of volume overload in decompensated heart failure. Circ Heart Fail 2009;2(5):499-504.

[14] Shin JT, Dec GW. Ultrafiltration should not replace diuretics for the initial treatment of acute decompensated heart failure. Circ Heart Fail 2009;2(5):505-11.

[15] Ministero della Salute. Raccomandazione n. 1 dell'anno 2008. Raccomandazione sul corretto utilizzo delle soluzioni concentrate di cloruro di potassio $-\mathrm{KCL}-\mathrm{e}$ altre soluzioni concentrate contenenti potassio. http://www.salute.gov.it/imgs/C_17_ pubblicazioni_584_allegato.pdf

[16] Allen LA, Turer AT, Dewald T, Stough WG, Cotter G, O'Connor CM. Continuous versus bolus dosing of furosemide for patients hospitalized for heart failure. Am J Cardiol 2010;105(12):1794-7.

[17] Felker GM, Lee KL, Bull DA, Redfield MM, Stevenson LW, Goldsmith SR, et al., NHLBI Heart Failure Clinical Research Network. Diuretic strategies in patients with acute decompensated heart failure. N Engl J Med 2011;364(9):797-805.

[18] Fonarow GC. Comparative effectiveness of diuretic regimens. $N$ Engl J Med 2011;364(9):877-8. 
[19] Drazner MH, Palmer BF. Hypertonic saline: a novel therapy for advanced heart failure? Am Heart J 2003;145(3):377-9.

[20] Taylor SH. Refocus on diuretics in the treatment of heart failure. Eur Heart J 1995;16(Suppl F):7-15.

[21] Brunton LL, Lazo JS, Parker KL (a cura di). Goodman \& Gilman. Le basi farmacologiche della terapia. XI Ed. Milabo: McGrawHill, 2006.

[22] Lee WH, Packer M. Prognostic importance of serum sodium concentration and its modification by converting-enzyme inhibition in patients with severe chronic heart failure. Circulation 1986;73(2):257-67.

[23] Sgambato F, Prozzo S. Le iponatriemia: problemi diagnostici e terapeutici, semplici e complessi. G It Medicina Interna 2003;2 Suppl(2):8-37.

[24] Brater DC. Resistance to loop diuretics. Why it happens and what to do about it. Drugs 1985;30(5):427-43.

[25] Vasko MR, Cartwright DB, Knochel JP, Nixon JV, Brater DC. Furosemide absorption altered in decompensated congestive heart failure. Ann Intern Med 1985;102(3):314-8.

[26] Brater DC. Diuretic resistance: mechanisms and therapeutic strategies. Cardiology 1994;84(Suppl 2):57-67.

[27] Ellison DH. Diuretic resistance: physiology and therapeutics. Semin Nephrol 1999;19(6):581-97.

[28] Paterna S, Di Pasquale P, Parrinello G, Fornaciari E, Di Gaudio F, Fasullo $S$, et al. Changes in brain natriuretic peptide levels and bioelectrical impedance measurements after treatment with high-dose furosemide and hypertonic saline solution versus high-dose furosemide alone in refractory congestive heart failure: a double-blind study. J Am Coll Cardiol 2005;45(12):19972003.

[29] Felker GM, O'Connor CM, Braunwald E, Heart Failure Clinical Research Network Investigators. Loop diuretics in acute decompensated heart failure: necessary?. Evil?. A necessary evil? Circ Heart Fail 2009;2(1):56-62.

[30] Salvador DR, Rey NR, Ramos GC, Punzalan FE. Continuous infusion versus bolus injection of loop diuretics in congestive heart failure. Cochrane Database Syst Rev 2005;(3):CD003178.

[31] Arnold JM, Howlett JG, Dorian P, Ducharme A, Giannetti N, Haddad $\mathrm{H}$, et al. Canadian Cardiovascular Society Consensus Conference recommendations on heart failure update 2007: Prevention, management during intercurrent illness or acute decompensation, and use of biomarkers. Can J Cardiol 2007; 23(1):21-45.

[32] Barracca A. L'acqua corporea e il sodio: un equilibrio perfetto. Fisiopatologia e alterazioni dell'equilibrio idroelettrolitico. Milano: Wichtig; 2006.

[33] Sgambato F. Il mare che è in noi. Atti del XVI Seminario "Gli equilibri in Medicina Interna. La patologia dell'Area Critica. Associazione Scientifica Incontri al Fatebenefratelli.(Benevento, 11-13 giugno 2009). 